\title{
Cardiac arrest in the toilet: clinical characteristics and resuscitation profiles
}

\author{
Joji Inamasu • Satoru Miyatake
}

Received: 2 February 2012/Accepted: 14 August 2012/Published online: 9 September 2012

(C) The Japanese Society for Hygiene 2012

\begin{abstract}
Objectives The great majority of non-traumatic cardiac arrests (CA) occur at home. The toilet is a closed and private room where CA occurs frequently. However, due to the feelings of privacy that are associated with this room, the circumstances and causes of CA in the toilet have rarely been investigated.

Methods A retrospective study was conducted to clarify clinical characteristics and resuscitation profiles of patients sustaining CA in the toilet.

Results Among 907 CA patients treated during a 4-year period, $101(11 \%)$ sustained CA in the toilet. While the collapse was witnessed in only $10 \%$ of these patients, return of spontaneous circulation (ROSC) was achieved in $41 \%$. However, the long-term survival rate was $1 \%$. Multivariate regression analysis revealed that a history of cardiac diseases was predictive of CA in the toilet (odds ratio 3.045; $95 \%$ confidence interval $1.756-5.282$ ) but that there was no correlation with advanced age. The frequency of CA in the toilet may be influenced moderately by seasonal/circadian variations. The 101 patients were classified into four subgroups according to mode of discovery of CA. The frequency of ROSC was highest in those who collapsed in the presence of caregivers and lowest in those whose collapse were discovered later by family members being worried that the patient stayed in the toilet "too long." Imaging studies revealed life-threatening extra-cardiac lesions responsible
\end{abstract}

\section{J. Inamasu $(\bowtie)$}

Department of Emergency Medicine, Fujita Health University

Hospital, 1-98 Kutsukake, Toyoake, Aichi 470-1192, Japan

e-mail: inamasu@fujita-hu.ac.jp

J. Inamasu $\cdot$ S. Miyatake

Department of Emergency Medicine,

Saiseikai Utsunomiya Hospital, Utsunomiya, Tochigi, Japan for CA, such as subarachnoid hemorrhage and aortic dissection, in $23 \%$ of the patient cohort.

Conclusions The rarity of long-term survival among individuals sustaining CA in the toilet is mainly due to the delay in discovering the individual who collapsed. Although a history of cardiac diseases is a risk factor, predicting who may sustain CA in the toilet remains difficult due to etiological heterogeneity.

Keywords Cardiac arrest - Return of spontaneous circulation $\cdot$ Toilet $\cdot$ Witnessed

\section{Introduction}

The great majority of non-traumatic cardiac arrests (CA) occur at home rather than in the commercial area or outdoors [1], and certain daily activities at home, such as taking a bath and going to the toilet (room), may trigger CA $[2,3]$. Toilets and bathrooms (containing the bathtub) are particularly unique from the perspective of resuscitation medicine because they are private, closed spaces, and the early detection of collapsed patients is usually difficult. Much attention has have been paid to CA that occur in the bathtub, particularly in Japan [4]. By contrast, fewer researchers have focused on patients sustaining $\mathrm{CA}$ in the toilet. Here, we report the results of a study conducted to clarify the clinical characteristics and resuscitation profiles of patients who sustained CA while in the toilet. The differences between sustaining $\mathrm{CA}$ in the toilet and in the bathroom (bathtub) are also discussed.

\section{Subjects and methods}

This is a retrospective study conducted in a single teaching hospital, which is also a tertiary referral center that covers a 
local population of approximately 500,000. The research protocol was approved by the institutional ethical committee. The observation period was between January 2006 and December 2009. In our hospital, cardiopulmonary resuscitation (CPR) for patients sustaining non-traumatic $\mathrm{CA}$ is performed in accordance with the recent basic/ advanced life-support guidelines [5], and prehospital data are documented in the Utstein style [6]. An electrocardiogram is obtained from the patient by emergency medical services at the scene or during his/her transfer. During CPR in our emergency department (ED), cardiac and abdominal ultrasonography are performed whenever possible to search for an extra-cardiac lesion that may be responsible for CA. In addition, a computed tomography (CT) scan of the whole body is obtained for those who achieve return of spontaneous circulation (ROSC), as long as their vitals signs are stable. Forensic autopsy to verify the cause of death of non-traumatic CA patients is rarely performed in our institution. Similarly, toxicological screening for drug overdose or substance abuse is not performed routinely for non-traumatic CA patients.

Information on where, when, and how CA occurred in each patient enrolled in our study had been obtained from family members or relatives who lived with the patient and was recorded in an institutional database by emergency medicine residents. We retrieved pertinent data both from both the database and medical charts. Only adult patients ( $\geq 20$ years of age) who sustained CA at home or nursing homes were included in the analysis.

\section{Statistical analysis}

Multivariate logistic regression analysis was conducted to identify clinical variables predictive of CA in the toilet. For the two-group comparison, the chi-square/Fisher exact test was used for categorical variables and the unpaired $t$ test for numerical variables. SPSS ver. 10.0 (SPSS, Chicago, IL) was used for the statistical analysis. Numerical data are presented as the mean \pm standard deviation (SD), and $P<0.05$ was considered to be statistically significant.

\section{Results}

\section{Demographics}

During the 4-year observation period of this study, 907 non-traumatic CA patients who sustained CA at home or at nursing homes were brought to our ED. Of these, 101 patients $(11 \%)$ sustained CA while in the toilet. Other compartments where the CA occurred were the bed/bedroom $(n=301,33 \%)$, living room $(n=179,20 \%)$, bathtub/shower room $(n=115,13 \%)$, dining room/ kitchen $(n=98,11 \%)$, and unspecified $(n=113,12 \%)$. The demographics and resuscitation profiles of the 907 patients are summarized in Table 1. Their age ranged from 22 to 99 years (mean $74.2 \pm 14.0$ years) and the male:female ratio was 5:4. The CA was witnessed in $40 \%$ of cases, ROSC was achieved in $33 \%$ of patients, and longterm survival (>12 months) was achieved by $3 \%$ of patients.

\section{Clinical characteristics of CA in the toilet}

The demographics and resuscitation profiles of the 101 patients who sustained CA while in the toilet are summarized in Table 2. Their age ranged from 26 to 96 years (mean $75.1 \pm 13.9$ years). Witnesses to the collapse were present in $10 \%$ of events, and ROSC was achieved in $41 \%$ of patients. One patient ( $1 \%)$ achieved a long-term survival of $>12$ months.

Table 1 Clinical characteristics and resuscitation profiles of the 907 patients who sustained cardiac arrest at home during the 4-years observation period

\begin{tabular}{ll}
\hline $\begin{array}{l}\text { Demographic/clinical } \\
\text { variables }\end{array}$ & Data \\
\hline Age (years) & $22-98$ (mean 74.2 \pm 14.0) \\
Male:female & $506: 401$ \\
Compartment where CA occurred & Toilet 101, bathtub/shower 115, kitchen/dining 98, bed(room) 301, living(room) 179, not specified 113 \\
History of cardiac diseases & $317(35 \%)$ \\
Diabetes mellitus & $203(22 \%)$ \\
Initial rhythm assessed by EMS & VF 74, non-shockable 833 \\
Witness to CA & $363(40 \%)$ \\
ROSC & $296(33 \%)$ \\
Long-term survival & $30(3 \%)$
\end{tabular}

Data are presented as the number of patients, with the percentage in parenthesis unless indicated otherwise

$C A$ Cardiac arrest, EMS emergency medical services, ROSC return of spontaneous circulation, $V F$ ventricular fibrillation 
Table 2 Demographics of 101 patients who sustained cardiac arrest in the toilet

\begin{tabular}{ll}
\hline Demographic/clinical variables & Data \\
\hline Age (years) & $26-96$ (mean 75.1 \pm 13.9$)$ \\
Male:female & $59: 42$ \\
History of cardiac diseases & $39(39 \%)$ \\
History of diabetes mellitus & $21(21 \%)$ \\
Initial rhythm & VF 8, non-shockable 93 \\
Witnesses of collapse & $10(10 \%)$ \\
ROSC & $41(41 \%)$ \\
Long-term survival & $1(1 \%)$ \\
\hline
\end{tabular}

Data are presented as the number of patients, with the percentage in parenthesis unless indicated otherwise

Frequency of witnessed collapse/ROSC and types of compartment

Whether the frequency at which the collapse was witnessed and/or whether ROSC was influenced by the type of compartment were investigated in 794 patients (following exclusion of the 113 patients for whom the location of the collapse was not specified). The frequency of the collapse being witnessed in the toilet $(10 \%)$ was not significantly higher than that in the bathroom (bathtub/shower; $8 \%$, $P=0.77$ ) (Fig. 1a). On the other hand, the frequency of ROSC in patients sustaining CA while in the toilet (41\%) was significantly higher than that in the bath room (bathtub/shower; $13 \%, P<0.01$ ) (Fig. 1b).

Multivariate regression analysis to identify risk factors

The demographic variables evaluated were age, sex, and comorbidities (history of cardiac diseases and diabetes mellitus). We found that the history of cardiac diseases was predictive of CA while in the toilet (odds ratio 3.045; $95 \%$ confidence interval 1.756-5.282; $P=0.002$ ). By contrast, none of the other variables were correlated with the occurrence of $\mathrm{CA}$ in patients during the time they were in the toilet (Table 3).

\section{Seasonal/circadian variation and CA in the toilet}

The 101 patients who sustained CA while in the toilet were classified into four seasonal quartiles (January-March, April-June, July-September, and October-December). The number of CA occurring in each quartile was 27, 22, 25, and 27, respectively (Fig. 2a). The 101 patients were subsequently stratified into four 6-h quartiles of the day $(0-6,6-12,12-18$, and $18-0 \mathrm{~h})$ depending on the time that the patient sustaining the $\mathrm{CA}$ was discovered (Fig. 2b). The number of CA in each quartile was 17, 30,
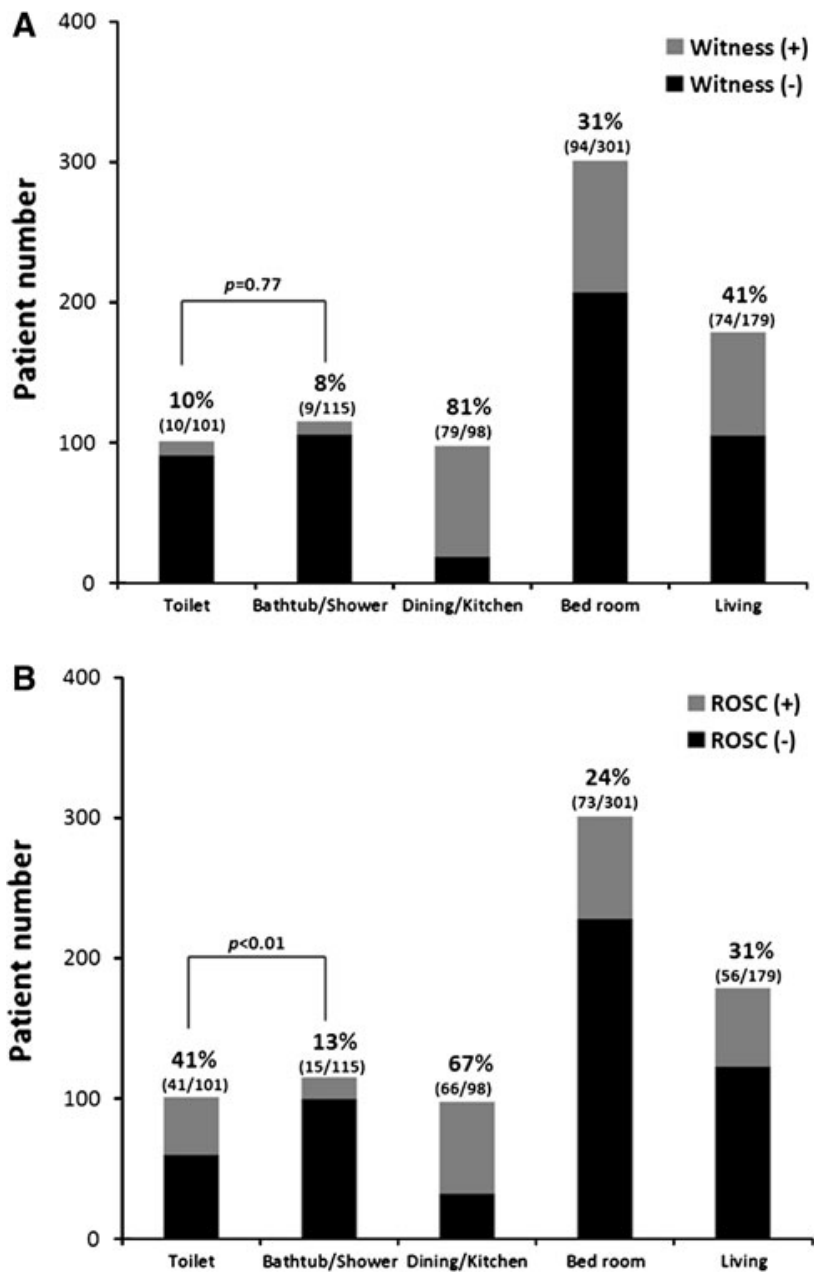

Fig. 1 Whether the frequency of the collapse was witnessed and/or whether the return of spontaneous circulation $(R O S C)$ were affected by the type of compartment was investigated in 794 patients (113 patients for whom the location of the collapse was not specified were excluded). a The frequency of the collapse being witnessed while the patient was in the toilet was not significantly higher than that in the bathtub/shower $(P=0.77)$. b The frequency of ROSC in patients sustaining $\mathrm{CA}$ while in the toilet was significantly higher than that in the bathtub/shower $(P<0.01)$

Table 3 Multivariate regression analysis to identify risk factors of cardiac arrest in the toilet

\begin{tabular}{llll}
\hline Demographic variables & Odds ratio & $\begin{array}{l}95 \% \text { confidence } \\
\text { interval }\end{array}$ & $P$ \\
\hline Age & 1.007 & $0.986-1.028$ & 0.502 \\
Male sex & 1.278 & $0.740-2.206$ & 0.379 \\
$\begin{array}{l}\text { History of cardiac } \\
\quad \text { diseases }\end{array}$ & 3.045 & $1.756-5.282$ & $0.002 *$ \\
Diabetes mellitus & 1.523 & $0.779-2.979$ & 0.219 \\
\hline
\end{tabular}

* Statistically significant

27 , and 27, respectively. Because of small number of CA in each time period, a statistical comparison could not be performed. 
Fig. 2 a The 101 patients who sustained cardiac arrest $(C A)$ in the toilet were stratified into four seasonal quartiles ( $x$-axis). The number of CA in each quartile was $27,22,25$, and 27 , respectively. b The 101 patients were stratified into four 6-h quartiles ( $x$-axis) depending on the time that the patient sustaining the CA was discovered. The number of CA in each quartile was $17,30,27$, and 2 , respectively

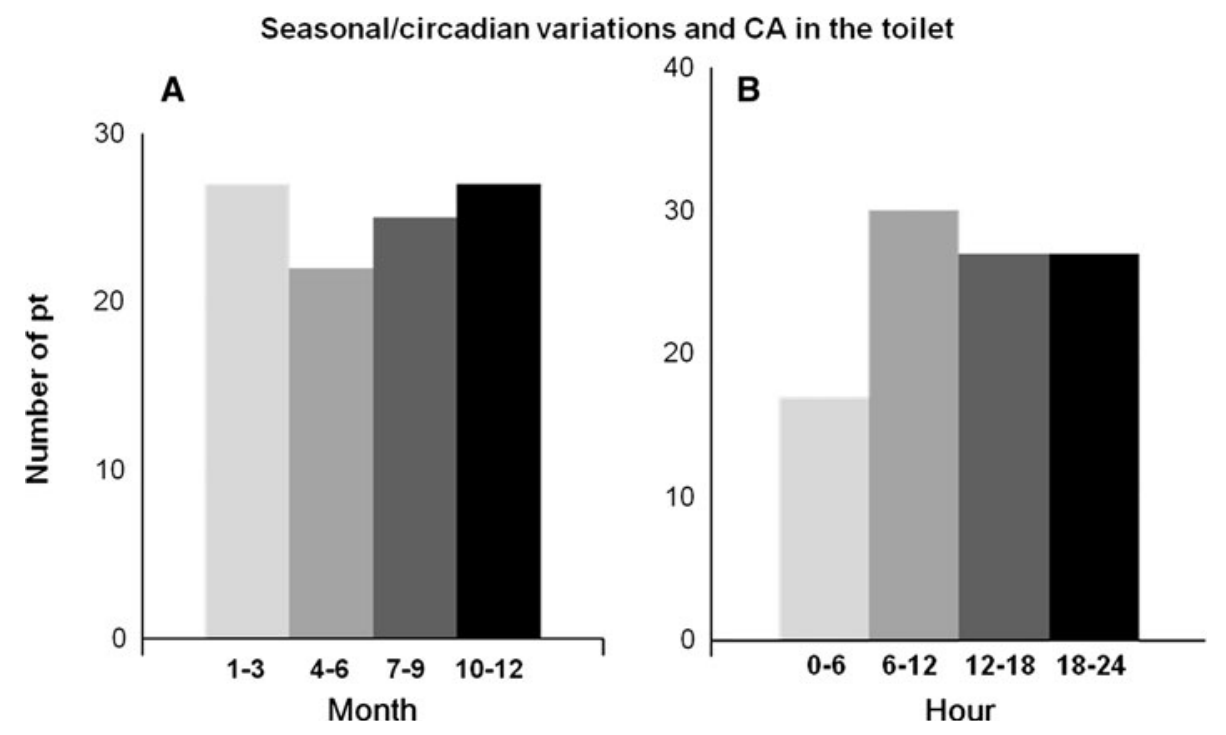

Mode of discovery of CA in the toilet

How the patient who sustained CA while in the toilet was discovered is summarized in Fig. 3. In 63 (62\%) events, the concern of family members that a patient had stayed in the toilet "too long" led to their visit to the toilet and subsequent discovery that the individual had sustained CA. In $22(22 \%)$ events, a loud sound/noise due to the patient's collapse onto the floor of the toilet prompted family members to investigate. In ten $(10 \%)$ events, the CA occurred in the presence of caregivers as the patient had been dependent on the help of caregivers and required assistance in the toilet. The only long-term survivor belonged to this subgroup. The remaining six patients $(6 \%)$ called or cried for help and were found on the floor by family members who rushed to the toilet. The rates of ROSC in these four subgroups were 30, 55, 70, and $57 \%$, respectively (Fig. 3). There were no significant intergroup differences in the frequency of ROSC. In none of the 101 patients was alarm call system available or deployed.

\section{Search for an extra-cardiac lesion as a cause of CA}

Cardiac and abdominal ultrasonography obtained during CPR in 69 patients $(68 \%)$ identified three patients with a ruptured abdominal aortic aneurysm (AAA), five patients in which the presence of a massive pericardial effusion and/or intimal flap of the aorta pointed to the diagnosis of aortic dissection (AD), and two patients who were diagnosed with a pulmonary embolism (PE). Among the 41 patients who achieved ROSC, 25 were hemodynamically stable enough to undergo a whole body CT, which
Mode of discovery of CA in the toilet and ROSC

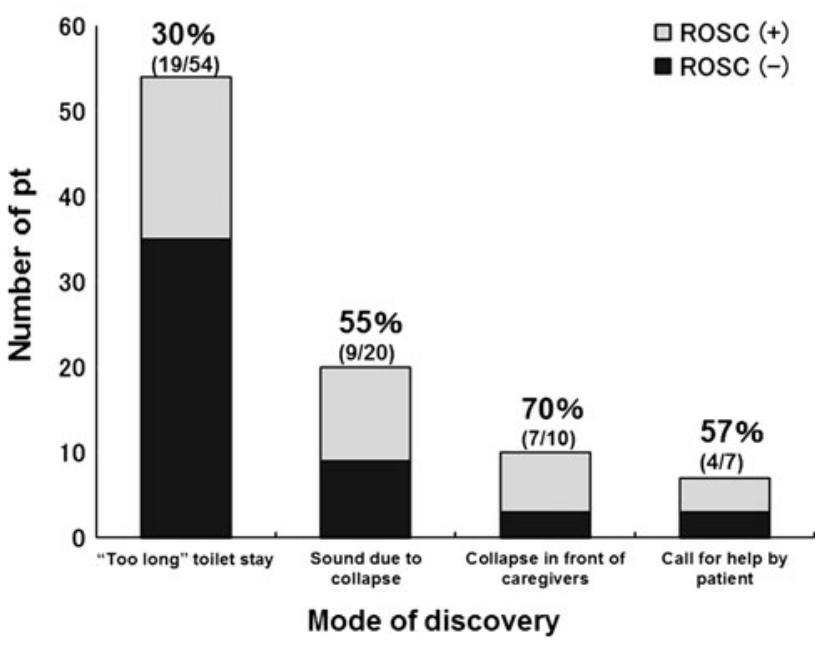

Fig. 3 How CA in the toilet was discovered is summarized in 101 patients. In 63 events (left), a concern that a patient stayed in the toilet too long led to the discovery; in 22 events (second from left), a loud sound/noise due to the patient's collapse onto the floor led to the discovery; in 10 events (third from left), CA occurred in the presence of caregivers. In the remaining 6 patients (right), they called for help from the toilet by themselves. There was no significant difference in the frequency of ROSC among the four groups

revealed eight patients with a subarachnoid hemorrhage (SAH) and three other patients with a multi-lobar pneumonia. Two other patients collapsed while in the toilet after sustaining a massive gastrointestinal bleeding. Therefore, at least 23 patients $(23 \%)$ sustained an extra-cardiac lesion that played a causative role in the CA that the patient sustained while in the toilet (Table 4). 

cause of cardiac arrest in the toilet revealed by imaging studies
Table 4 Extra-cardiac lesion as

\begin{tabular}{lr}
\hline Etiology & $n$ \\
\hline Subarachnoid hemorrhage & 8 \\
Aortic dissection & 5 \\
Ruptured abdominal aortic & 3 \\
$\quad$ aneurysm & \\
Multi-lobar pneumonia & 3 \\
Gastrointestinal tract & 2 \\
bleeding & \\
Pulmonary embolism & 2 \\
Total & 23 \\
\hline
\end{tabular}

\section{Discussion}

Most people spend a relatively short time per day in the toilet: according to a Japanese study, this amounts to an average of $30 \mathrm{~min}(2 \%)$ daily [2]. Nevertheless, the toilet seems to be a location where CA occurs with a disproportionately high frequency: $8-10 \%$ of all CA may occur in individuals while they are in the toilet $[2,3]$. The toilet and bathroom (bathtub) are particularly unique from the perspective of resuscitation medicine because they are closed, private spaces, and the early detection patient collapse is usually difficult. The results of this study, in which the frequency of CA in the toilet was $11 \%$, are comparable with those reported in the literature $[2,3]$. The similarity between $\mathrm{CA}$ in the toilet and bathroom (bathtub) is that frequency of the collapse being witnessed is low (10 vs. $8 \%$, respectively; Fig. 1a). On the other hand, the frequency of ROSC was significantly lower in the latter (41 vs. $13 \%$; Fig. 1b). Patients are often drowned and/or become hyperthermic in the bathtub by the time rescue efforts are initiated [4], which may explain the poorer outcomes in the latter. However, despite the seemingly better resuscitation profiles represented by the high ROSC rate, the long-term outcomes of patients sustaining $\mathrm{CA}$ while in the toilet were poor in our study, with a survival rate of $1 \%$.

We also investigated whether the mode of discovery of the individual sustaining the CA in the toilet affected the resuscitation profiles and outcomes. Collapse was witnessed only in those who required assistance in toilet use because of preexisting physical disabilities, and it is natural that the frequency of ROSC was highest in this subgroup (Fig. 3). However, these individuals suffered from debilitating illnesses and had limited health reserves; consequently, the high ROSC rate might not have translated into improved outcomes. In contrast, the frequency of ROSC was lowest in those who stayed in the toilet "too long," and the frequencies of ROSC in the other two groups were intermediary. These results indicate the difficulty of early detection of individuals sustaining CA while in the toilet: while more than $60 \%$ of patients collapsed without being noticed by family members, only $6 \%$ were able to call for help by themselves. In none of the 101 patients was an alarm call system available or used. Even if such alarm call system had been available, however, its efficacy may have been limited [7] because only a fraction of patients would have been able to activate the alarm call system before collapse.

Another factor we investigated was whether the frequency of individuals sustaining CA while in the toilet was affected by demographic variables. The results of the multivariate regression analysis (Table 3) revealed, as expected, that a history of cardiac disease was a risk factor. Contrary to our expectations, however, advanced age was not correlated with an elevated frequency of $\mathrm{CA}$ in the toilet. The occurrence of $\mathrm{CA}$ is known to be affected by seasonal or circadian variation: in the northern hemisphere, $\mathrm{CA}$ is less likely to occur in the spring to summer seasons [8] and during the early morning hours (0-6 h) [9]. Our results (Fig. 2a, b) seem to be compatible with those reported in the literature $[8,9]$, suggesting that $\mathrm{CA}$ in the toilet may also be affected by seasonal/circadian variations. Because of the small number in each subgroup and the lack of statistical analysis, however, this theory remains speculative.

There is little doubt that the causes of CA in the toilet are heterogenous. With the use of imaging studies, a substantial number of extra-cardiac lesions, such as SAH, AD, PE, and ruptured AAA, were identified in our patient cohort (Table 4). Most of these were vascular diseases that may be triggered by the sudden rise in blood pressure that occurs during the Valsalva maneuver associated with the act of defecation/micturition. In fact, defecation/micturition was found to be a triggering event in $13 \%$ of patients with $\mathrm{SAH}$, a relatively common cause of $\mathrm{CA}[10,11]$. AD may also be triggered by straining to empty the bowel/bladder: defecation/micturition was reported to be a triggering event in $8 \%$ of patients with AD [12]. Similarly, PE seems to be causally associated with defecation/micturition [13]. It is probable that the true incidence of extra-cardiac lesions as the cause of CA that occurs when an individual is actually sitting on the toilet might be higher than the current figure of $23 \%$, since ultrasonography, a useful imaging modality for identifying the causes of CA [14], was performed on less than $70 \%$ of our patient cohort. On the other hand, the great majority of patients who sustain CA while in the toilet may not harbor an extra-cardiac lesion. Our multivariate regression analysis (Table 3 ) suggests that these patients may have sustained acute coronary syndrome and/or ventricular arrhythmia during or immediately after defecation/micturition [2, 3]. Several authors believe that the occurrence of CA on the toilet is the consequence of severe defecation/micturition syncope [15]. In patients with defecation/micturition syncope, a paradoxical decrease in blood pressure during straining occurs due to imbalance between sympathetic and 
parasympathetic autonomic function, which may in turn cause decreased cerebral blood flow and loss of consciousness [15-18]. Both increased extra-cardiac vascular events after suddenly elevated blood pressure and increased cardiac events after imbalance between sympathetic and parasympathetic nerves may be responsible for the disproportionally high frequency of CA while an individual is in the toilet.

There are several limitations to this study. First, for most patients it is generally unclear whether they collapsed after defecation or micturition, although defecation usually requires stronger straining forces and may be more frequently associated with CA. Patient demographics between defecation and micturition syncope seem to differ substantially [19]. Second, it is possible that several patients might have visited the toilet after feeling sick or nauseated and collapsed there: therefore, their collapse in the toilet might not necessarily be related to the act of defecation or micturition. For example, impending rupture of an AAA may provide the urge for patients to urinate because the bladder is compressed by the aneurysm [20]. Similarly, patients who feel sick and go to the toilet may have better chance of being transferred to ED compared with those who collapse in other areas: such differential bias also has to be considered. Third, we were unable to evaluate the influence of medication because of limited data. Therefore, the possibility that the CA might have been due to the side effects of an anti-hypertensive, oral hypoglycemic agent, or insulin can not be excluded. Finally, this is not an epidemiological study. CA occurring in individuals who live alone may go unnoticed for a long time, and these individuals may actually die before receiving attention. Despite such limitations, however, this is the one of the first studies to evaluate the clinical characteristics and resuscitation profiles of individuals sustaining $\mathrm{CA}$ in the toilet. Although the prediction of who may sustain CA in the toilet remains difficult, we expect that measures to prevent or reduce CA in the toilet will be available in the future.

Conflict of interest There is no financial relationship with any biotechnology manufacturer, pharmaceutical company, or other commercial entity that has an interest in the subject matter or materials discussed in the manuscript.

\section{References}

1. Folke F, Gislason GH, Lippert FK, Nielsen SL, Weeke P, Hansen ML, et al. Differences between out-of-hospital cardiac arrest in residential and public locations and implications for publicaccess defibrillation. Circulation. 2010;122:623-30.

2. Hayashi S, Toyoshima H, Tanabe N, Satoh T, Miyanishi K, Seki $\mathrm{N}$, et al. Activity immediately before the onset of non-fatal myocardial infarction and sudden cardiac death. Jpn Circ J. 1996; 60:947-53.

3. Matoba R, Shikata I, Iwai K, Onishi S, Fujitani N, Yoshida K, et al. An epidemiologic and histopathological study of sudden cardiac death in Osaka Medical Examiner's Office. Jpn Circ J. 1989;53:1581-8.

4. Shigeomi S, Sato W, Enzan K, Yoshioka N. Sudden death during bathing in the elderly. J Jpn Assoc Acute Med. 2001;12:109-20.

5. International Liaison Committee on Resuscitation. International consensus on cardiopulmonary resuscitation and emergency cardiovascular care science with treatment recommendations. Part 4: advanced life support. Resuscitation. 2005;67:213-47.

6. Langhelle A, Nolan J, Herlitz J, Castren M, Wenzel V, Soreide E, et al. Utstein consensus symposium. Recommended guidelines for reviewing, reporting, and conducting research on post-resuscitation care: the Utstein style. Resuscitation. 2003;205(66): 271-83.

7. Monro A, Mulley GP. Hospital bathrooms and showers: a continuing saga of inadequacy. J R Soc Med. 2004;97:235-7.

8. Arntz HR, Willich SN, Schreiber C, Brüggemann T, Stern R, Schultheiss HP. Diurnal, weekly and seasonal variation of sudden death. Population-based analysis of 24,061 consecutive cases. Eur Heart J. 2000;21:315-20.

9. Peckova M, Fahrenbruch CE, Cobb LA, Hallstrom AP. Circadian variations in the occurrence of cardiac arrests: initial and repeat episodes. Circulation. 1998;98:31-9.

10. Matsuda M, Watanabe K, Saito A, Matsumura K, Ichikawa M. Circumstances, activities, and events precipitating aneurysmal subarachnoid hemorrhage. J Stroke Cerebrovasc Dis. 2007;16: 25-9.

11. Inamasu J, Miyatake S, Tomioka H, Suzuki M, Nakatsukasa M, Maeda N, et al. Subarachnoid haemorrhage as a cause of out-ofhospital cardiac arrest: a prospective computed tomography study. Resuscitation. 2009;80:977-80.

12. Kojima S, Sumiyoshi M, Nakata Y, Daida H. Triggers and circadian distribution of the onset of acute aortic dissection. Circ J. 2002;66:232-5.

13. Yamada N, Nakamura $M$, Ishikura $K$, Ota $M$, Yazu T, Ota $S$, et al. Triggers of acute pulmonary thromboembolism developed in hospital, with focusing on toilet activities as triggering acts. Int J Cardiol. 2005;98:409-11.

14. Hernandez C, Shuler K, Hannan H, Sonyika C, Likourezos A, Marshall J. C.A.U.S.E.: cardiac arrest ultra-sound exam-a better approach to managing patients in primary non-arrhythmogenic cardiac arrest. Resuscitation. 2008;76:198-206.

15. Kapoor WN, Peterson J, Karpf M. Defecation syncope. A symptom with multiple etiologies. Arch Intern Med. 1986;146: 2377-9.

16. Culić V. Triggering of cardiovascular incidents by micturition and defecation. Int J Cardiol. 2006;109:277-9.

17. Sikirov BA. Cardio-vascular events at defecation: are they unavoidable? Med Hypotheses. 1990;32:231-3.

18. Allan L, Johns E, Doshi M, Anne Kenny R, Newton JL. Abnormalities of sympathetic and parasympathetic autonomic function in subjects with defaecation syncope. Europace. 2004;6: $192-8$.

19. Komatsu K, Sumiyoshi M, Abe H, Kohno R, Hayashi H, Sekita $\mathrm{G}$, et al. Clinical characteristics of defecation syncope compared with micturition syncope. Circ J. 2010;74:307-11.

20. Hadjis NS, McAuley GJ, Ruo L, Kwauk S, Olatunbosun F. Acute abdominal pain and urgency to defecate in the young and the old: a useful symptom-complex? J Emerg Med. 1999;17:239-42. 